\title{
Topical tretinoin: its use in daily practice to reverse photoageing
}

\author{
M.T.GOLDFARB, C.N.ELLIS AND J.J.VOORHEES \\ Dermatopharmacology Unit, Department of Dermatology, University of Michigan Medical Center, Ann Arbor, \\ Michigan, U.S.A.
}

\section{SUMMARY}

The effect of $0 . \mathbf{I} \%$ tretinoin cream for the treatment of photoageing was studied in a doubleblind, placebo-controlled trial. All patients applied tretinoin cream to one forearm and the vehicle cream to the other, and half of the patients also applied tretinoin cream to the face and the other half used the vehicle cream. Tretinoin treatment produced an improvement in the signs of extrinsic ageing compared with the vehicle-treated areas. Fine wrinkling was improved most, although coarse wrinkling, brown spots, tactile roughness and overall skin colour also showed clear improvement. The majority of lentigines and sun-induced freckles showed some reduction in coloration with extended treatment. It is important when using tretinoin that the treatment procedure is carefully explained to the patients and that they are warned about a retinoid reaction. It should be stressed that improvement is gradual and that regular application of the cream must continue even after improvement has been achieved. Patients should be assured that there is no evidence of carcinogenicity in humans. Although no teratogenic effects of tretinoin have been reported when applied topically, it is not advisable to use the cream when trying to conceive or when pregnant.

Tretinoin (all-trans-retinoic acid) has been used topically for the treatment of acne for the last two decades. More recently, it has been successfully tested and prescribed for the reversal of photoageing ${ }^{1,2}$ but, in order to achieve significant improvement using topical tretinoin, patients must be carefully educated in how to apply the medication and on the degree of improvement that they can expect. An overview of the use of topical tretinoin in photoageing is presented and how patients should be instructed on the proper use of this medication is considered.

\section{TRETINOIN: MECHANISM OF ACTION}

Although the exact mechanism of action of tretinoin is unknown, numerous studies have been conducted in animals and man on its effects on the skin. Tretinoin can lead to many changes in

Correspondence: Professor J.J.Voorhees, Department of Dermatology, University of Michigan Medical Center, I 500 East Medical Center Drive, I9I0 Taubman Center, Ann Arbor, MI 48109-0314, U.S.A. 
the epidermis, including increased keratinocyte proliferation, ${ }^{3}$ increased keratohyalin granule formation, ${ }^{4}$ decreased keratinocyte cohesion, ${ }^{5}$ decreased melanocyte activity ${ }^{6}$ and altered keratin composition, ${ }^{5}$ as well as production of mucin. ${ }^{4}$ In the dermis of mice, tretinoin induces the formation of new collagen and blood vessels. ${ }^{1}$ It is easy to assume that the alterations induced by tretinoin would be beneficial in the treatment of thin, wrinkled, yellow skin characteristic of photoageing.

Tretinoin may exert its effects on the cell through a specific nuclear receptor: the human retinoic acid receptor. ${ }^{7}$ After binding tretinoin, the receptor is activated and can then interact with cellular DNA, leading to an alteration in gene expression, which may result in structural and functional changes.

\section{TRETINOIN: PREVIOUS EXPERIENCE IN PHOTOAGEING}

The reversal of photoageing and wrinkling of the skin by tretinoin was first noted in patients treated for actinic comedones; they reported resolution of the comedones and smoother, less wrinkled skin. Studies on hairless, albino mice have demonstrated that the dermal damage induced by UV radiation could be partially reversed by applying tretinoin topically, but not by applying a bland cream or non-specific irritants. ${ }^{8}$

One of the first studies on the use of topical tretinoin for the reversal of photodamage in man focused on the histological structure and function of the skin. ${ }^{1}$ Changes noted included an increase in epidermal thickness, a decrease in the atypia of the keratinocytes and a more even distribution of melanin granules, as well as an increased production of collagen and greater fibroblast activity in the dermis.

In a subsequent double-blind, placebo-controlled trial of tretinoin cream for the reversal of photodamage, patients received $0.1 \%$ tretinoin cream and a vehicle cream. ${ }^{2}$ They applied one cream to the face and left dorsal forearm and the other to the right dorsal forearm for 4 months. Assessment of treatment included clinical evaluations, photography and punch biopsies ( $4 \mathrm{~mm}$ ) taken from both dorsal forearms before and after treatment. Clinical improvement was noted in almost all tretinoin-treated areas, but not in those treated with vehicle. $A$ decrease in fine wrinkling was the most obvious change, followed by an increased pinkness, a smoother texture and less coarse wrinkling of the skin. Incidental findings were a lightening of hyperpigmented spots or lentigines and fewer actinic keratoses. Histological studies of the dorsal forearm showed a compact stratum corneum, a thicker epidermis and granular cell layer, reduced epidermal cell dysplasia, fewer melanocytes and the formation of mucin-like material in the stratum corneum with tretinoin therapy. Using electron microscopy, ${ }^{6}$ tretinoin-treated skin demonstrated less melanocyte activity, as well as an increase in anchoring fibrils and filaments at the epidermaldermal junction. These findings were not noted in the vehicle-treated skin.

Some of the patients participated in a further study on the extended use of topical tretinoin in photoageing. ${ }^{9}$ After using tretinoin for almost 2 years, there was an even greater improvement in fine and coarse wrinkling and a significant decrease in the number of discrete lentigines. Most of the improvement had been achieved after 6-10 months, with maintenance of the positive results and some additional benefit after longer use of tretinoin.

\section{TRETINOIN: USE IN PRACTICE}

The positive results of these clinical studies lead to many patients requesting a prescription for tretinoin cream to 'remove their wrinkles', many being under the false impression that the 
treatment would involve only a brief series of applications of the cream and that their wrinkles would rapidly begin to fade without any adverse reactions.

Various concentrations of tretinoin have been tried for the reversal of photoageing, but there is currently no consensus on the best one. There is evidence that the improvement in photoageing using tretinoin is dose-dependent and, therefore, the highest commercially available form, $0.1 \%$ cream, should give the best results. During the first 3 months of tretinoin therapy, patients may experience erythema, peeling, burning and itching of the skin; a similar reaction has also been noted using other retinoids given both topically and systemically. With higher concentrations of tretinoin, this reaction can be more pronounced and may affect more than $90 \%$ of patients. ${ }^{2}$ Some physicians, therefore, will primarily use $0.025 \%$ tretinoin cream but it remains to be proved if this lower dose of tretinoin, even when used over an extended period, can lead to the same degree of improvement as the higher concentrations. It is currently recommended that patients use the maximal dose of tretinoin that does not cause a significant retinoid reaction; a patient is applying the correct dose if there is some intermittent peeling and erythema.

In general, patients start the treatment by applying a thin coat of $0.1 \%$ tretinoin cream at night. They are instructed to rub the medication carefully into all areas of photoaged skin. Many patients tend to avoid treating the periorbital wrinkles because of the proximity to the eyes; however, these wrinkles respond the best to tretinoin therapy. The patient may have difficulty initially in applying the tretinoin every evening because of the retinoid reaction, which can begin after only 2 days and may quickly become quite uncomfortable. ${ }^{2}$ They should then be instructed to use the cream every second or third evening, which allows the skin to acclimatize slowly. Most patients find the retinoid reaction resolves after $2-12$ weeks. ${ }^{2}$ If the reaction becomes very irritating, a low-dose corticosteroid cream, such as $\mathrm{I} \%$ hydrocortisone, may be applied for a few days to hasten the resolution, and occasionally it may even be necessary for the patient to change temporarily to a lower concentration of tretinoin. Gradually, most patients are able to tolerate $0.1 \%$ tretinoin cream every evening and can apply more of it without any problems. Several thin coats of the cream in succession, rather than one thick coat, result in the greatest penetration of the medication. It is important that patients maintain the therapy because, if they discontinue, the retinoid reaction may recur when therapy is resumed.

It is imperative for the patient to understand that the retinoid reaction of tretinoin therapy is a side-effect that most will experience in the early stages of treatment and is not an allergy or reason to discontinue the medication completely. They should be told that there are two other reactions that lead to increased erythema of skin which are distinct from the retinoid reaction. ${ }^{2}$ One is the pleasing pink colour of the skin which develops early in tretinoin therapy without any associated peeling, burning or itching. The other reaction presents as small, discrete areas of erythema and scaling after a few months of therapy; these may be subclinical actinic keratoses that have become inflamed. This inflammation may be the first step to their complete resolution and is, therefore, desirable.

The cream formulation of tretinoin has been most frequently prescribed as it is less drying than the gel or solution and, therefore, is better suited for photoaged skin. It is best if the patient applies the medication at bedtime; daytime use is less desirable because tretinoin may be inactivated by the sun. The cream should not be applied with any other topical agents in order to prevent the tretinoin being diluted, as well as avoiding possible incompatibilities.

As there is occasional peeling and mild irritation of the skin with tretinoin therapy, even after many months of use, a daytime moisturizer is recommended. The scaly skin associated with tretinoin is best removed with a mild soap or a soap-free cleanser, using the fingertips. Wash 
cloths, sponges and abrasive agents should be avoided as they can traumatize the fragile stratum corneum of tretinoin-treated skin leading to superficial erosions.

Although the face is the most common area patients wish to treat, many also want to use tretinoin on the neck, upper chest, dorsal forearms and hands. Patients should be warned that these areas are more prone to develop a significant retinoid reaction and it may take longer for the skin to become acclimatized.

Prior to the initiation of therapy, patients will have a broad range of expectations and, if they believe that they will obtain the same degree of dramatic improvement that is achieved with face lifts and chemical peels, they will certainly be disappointed but, if they are properly instructed, most patients will be pleased with the improvement. During the first month of treatment, the patient may notice a pleasing pink colour and a tightening of the skin; it takes at least 2 months before the fine wrinkles and, to a lesser degree, the coarse wrinkles begin to fade. The lentigines will begin to lighten during the first few months, but it takes approximately 6 months for complete resolution. After 6 months or more of tretinoin therapy, some of the sagging skin, especially in the periorbital area, may begin to tighten. The greatest improvement will occur during the first 12 months of therapy ${ }^{9}$ with only minimal improvement thereafter. Discontinuing topical tretinoin will probably lead to a slow reversal of the response and patients must continue to apply the medication at least twice a week indefinitely to maintain positive results.

One problem with tretinoin therapy is documenting the improvement because the skin changes are gradual and the physician cannot rely on memory and a written clinical record. A professional photographer and a special camera unit are necessary to produce adequate photographs but this is usually possible only under a research protocol. The physician, however, may be able to note a pink tone and possibly an overall smoother texture and appearance of the skin by clinical examination and patients may report their own, and others', positive comments.

It has been reported that tretinoin therapy leads to an increased sensitivity of the skin to sun. In the authors' experience, after treating hundreds of patients, this has not been a significant problem. It is recommended, however, that a sunscreen with a protection factor of 15 or greater is applied every morning during tretinoin therapy. This eliminates the possible increased sensitivity to UV radiation and helps to prevent further photodamage. Patients who deliberately expose their skin to the sun are not candidates for tretinoin therapy as the continuing photodamage is likely to outweigh the positive effects possible from topical tretinoin.

Some patients have been told that tretinoin is carcinogenic as it had been demonstrated that topical tretinoin enhanced the formation of skin malignancies in hairless albino mice after treatment with a carcinogenic dose of UV radiation. ${ }^{10,11}$ Other experiments have not only refuted these results but have actually shown that the application of tretinoin can reduce the formation of such cancers. ${ }^{11}$ Further evidence against its carcinogenic effect is the fact that there have been no skin or other malignancies associated with the use of topical tretinoin in the millions of people treated over the last two decades. On the contrary, topical tretinoin is now being investigated as a possible treatment for premalignant and malignant skin conditions. Positive results with tretinoin therapy have already been reported for actinic keratoses, leukoplakia, basal cell carcinoma, dysplastic naevi and metastatic melanoma. ${ }^{12,13}$

It has been questioned whether tretinoin can be safely used during pregnancy. As with other retinoids, tretinoin can be teratogenic when used systemically, but even after applying large amounts of tretinoin to the skin there has been no evidence of the drug or its metabolites in the blood. ${ }^{14}$ Furthermore, even after years of using topical tretinoin for the treatment of acne in young women, there have been no reports of birth defects. ${ }^{15}$ An analysis of topical tretinoin use 
in the first trimester of pregnancy has not shown any increase in the frequency of birth defects or the type of defects noted with retinoid use. ${ }^{16}$ Despite this, it is not advisable to treat any patient with topical tretinoin who is trying to conceive or who is pregnant.

As there is a large amount of information for patients to absorb when initiating tretinoin therapy, it is helpful to provide them with a printed sheet explaining exactly how to use the medication and what to expect. This should not replace a discussion with each patient, but should be used as a helpful reference for them to consult at home. It is recommended that the patient return after I month of therapy, as many questions may have arisen by then; thereafter, patients should be followed at approximately 3 -month intervals if possible.

\section{CONCLUSION}

Topical tretinoin has been found to be effective in the treatment of photoageing in both research studies as well as in general clinical use, although some patients and physicians have reported suboptimal results. When patients have received exact instructions on how to use topical tretinoin and have had explained to them the degree of improvement that is possible with this therapy, results are frequently positive.

\section{ACKNOWLEDGMENT}

This was supported in part by the Babcock Dermatological Endowment.

\section{REFERENCES}

I Kligman AM, Grove GL, Hirose R, Leyden JJ. Topical tretinoin for photoaged skin. 7 Am Acad Dermatol 1986; I5: $836-59$.

2 Weiss JS, Ellis CN, Headington JT et al. Topical tretinoin improves photoaged skin: a double-blind, vehiclecontrolled study. F Am Med Assoc 1988; 259: 527-32.

3 Connor MJ, Lowe NJ. Retinoid stimulation of epidermal cell growth in vivo. In: Retinoids (Saurat J, ed). Basel: $\mathrm{S}$ Kruger, 1985; 198-20I.

4 Elias PM. Epidermal effect of retinoids: supramolecular observations and clinical implications. 7 Am Acad Dermatol 1986; 15: 797-809.

5 Eichner R. Epidermal effects of retinoids: in vitro studies. $\mathcal{F}$ Am Acad Dermatol 1986; r5: 789-97.

6 Zelickson AS, Mottaz JH, Weiss JS et al. Topical tretinoin in photoaging: an ultrastructural study. $\mathcal{F}$ Cutan Aging Cosmetic Dermatol I988; I: 4 I-7.

7 Petkovick M, Brand NJ, Drust A et al. A human retinoic acid receptor which belongs to the family of nuclear receptors. Nature I987; 330: $444-50$.

8 Kligman LH. Effects of all-trans-retinoic acid on the dermis of hairless mice. F Am Acad Dermatol 1986; 15:779-85.

9 Ellis CN, Weiss JS, Hamilton TA et al. Sustained improvement with prolonged topical tretinoin (retinoic acid) for photoaged skin. $\mathcal{J} A m$ Acad Dermatol (in press).

Io Epstein JH. Chemicals and photocarcinogenesis. Aust $\mathcal{F}$ Dermatol 1977; 18: 57-61.

I I Epstein JH. All-trans-retinoic acid and cutaneous cancers. F Am Acad Dermatol 1986; 15: 772-8.

12 Peck GL. Topical tretinoin in actinic keratosis and basal cell carcinoma. $\mathcal{F}$ Am Acad Dermatol 1986; 15: 829-35.

13 Meyskens FL. Role of topical tretinoin in melanoma and dysplastic nevi. 7 Am Acad Dermatol 1986; 15: 822-5.

I4 Chaing TC. Gas chomatographic-mass spectrometric assay for low levels of retinoic acid in human blood. $f$ Chromatogr 1980; 182: 335-40.

I5 Kligman AM. Is topical tretinoin teratogenic? F Am Med Assoc 1988; 259: 2918.

I6 FDA and teratogenic potential of Retin-A. Scrip I989; no. 1374:5-17. 
This document is a scanned copy of a printed document. No warranty is given about the accuracy of the copy. Users should refer to the original published version of the material. 